\title{
121. On a Property of Mappings of Metric Spaces
}

\author{
By Kiyoshi IsÉKı \\ Kobe University \\ (Comm. by K. KUnUGI, M.J.A., July 12, 1954)
}

In his paper, "Solid Spaces and Absolute Retracts" (Ark. Mat., 1, 375-382 (1952)), O. Hanner has proved that any metric NES (normal) is an absolute $G_{\delta}$.

In this note, we shall prove the following theorem:

Any metric NES (completely normal) is an absolute $G_{\delta}$.

Let $\alpha$ be a class of topological spaces, and $A$ a space of $\alpha$. The space $Y$ is called an NES ( $\alpha$ ), if every mapping $f$ of a closed subset $A$ of a space $X$ of $\alpha$ into $Y$ can be extended to a mapping $f^{\prime}$ of an open set $U$ into $Y$ such that $A \subset U \subset X$.

A space $X$ is called an absolute $G_{\delta}$, if whenever $X$ is topologically imbedded in a metric space $Y$, then $X$ is a $G_{\delta}$ in $Y$.

To prove the theorem, we shall use the method employed by O. Hanner.

Let $X_{1}$ be any metric space containing $X$, and $Z$ one to one with $X_{1}$. Let $h$ be the (1-1)-correspondence from $Z$ onto $X_{1}$. We shall introduce a topology in $Z$ by taking as open sets in $Z$

$$
h^{-1}(O) \cup A
$$

where $O$ is any open set of $X_{1}$ and $A$ is any set of $Z-h^{-1}(X)$. Then $h$ is continuous and $X^{\prime}=h^{-1}(X)$ is closed in $Z$.

The topological space $Z$ is completely normal. Let $A_{1}, A_{2}$ be separated sets in $Z$. Let $B_{i}=h\left(A_{i} \cap X^{\prime}\right)(i=1,2)$, then $B_{1}, B_{2}$ are separated in the metric space $X_{1}$. Therefore, the two sets $O_{1}=\{x$ $\left.\mid \rho\left(B_{1}, x\right)<\rho\left(B_{2}, x\right) \& x \in X_{1}\right\}, \quad O_{2}=\left\{x \mid \rho\left(B_{1}, x\right)>\rho\left(B_{2}, x\right) \& x \in X_{2}\right\}$ are disjoint open. Hence $U_{1}=h^{-1}\left(O_{1}\right) \cup A_{1}, U_{2}=h^{-1}\left(O_{2}\right) \cup A_{2}$ are disjoint open in $Z$, and $U_{i} \supset A_{i}(i=1,2)$. Thus $Z$ is completely normal.

To prove that $X$ is an absolute $G_{\delta}$, we shall use an argument of C. H. Dowker. ${ }^{\text {1) }}$

The partial mapping $h \mid X^{\prime} \rightarrow X$ is extended to a mapping $h^{\prime}$ of an open set $U$, such that $X^{\prime} \subset U \subset X$, into $X$, since $X$ is an NES (completely normal). Let

$$
f(x)=\rho\left(h(x), h_{1}(x)\right) \quad \text { for } \quad x \in U,
$$

then $f(x)$ is continuous, and $f(x)=0$ if and only if $x \in X^{\prime}$. This shows that $X^{\prime}$ is a $G_{\delta}$ in $U$. There are open sets $U_{n}$ in $U$ such that $X^{\prime}$ $=\bigcap_{n=1}^{\infty} U_{n}$. Hence every $U_{n}$ is open in $Z$, and $h\left(U_{n}\right)=V_{n} \cup A_{n}(n=1,2$, $\cdots)$ where $V_{n}$ is open in $X_{1}$ and $A_{n} \subset X_{1}-X$. Thus $X=\bigcap_{n=1}^{\infty} V_{n}$ and $X$ 
is a $G_{\delta}$ in $X_{1}$. This completes the proof.

In a recent note, ${ }^{2)}$ the present author introduced a notion, absolute neighborhood retract for the class of completely normal spaces and proved that $X$ is ANR (completely normal) if and only if it is NES (completely normal). Therefore, we have the following

Theorem. Any metric ANR (completely normal) is an absolute $G_{\delta}$, and hence topologically complete.

By my previous results ${ }^{3)}$ and similar method, we have the following

Theorem. Any metric ANR (countably paracompact) is an absolute $G_{\delta}$.

The theorem for paracompact spaces was proved by O. Hanner ((4), p. 333, Theorem 14.1).

Theorem. Any separable metric ANR (hypocompact) is an absolute $G_{\delta}$.

This is proved by a similar argument using a result of S. Kaplan ((5), p. 249).

\section{References}

1) C. H. Dowker: On a theorem of Hanner, Ark. Mat., 2, 307-313 (1952).

2) K. Iséki: On the Hannerisation of completely normal spaces, to appear soon.

3) K. Iséki: On Hannerisation of two, countably paracompact normal spaces, Proc. Japan Acad., 30, 443-444 (1954).

4) O. Hanner: Retraction and extension of mappings of metric and non-metric spaces, Ark. Mat., 2, 315-360 (1952).

5) S. Kaplan: Homology theory of arbitrary subsets of Euclidean spaces, Trans. Am. Math. Soc., 62, 248-271 (1947). 